\title{
A Suspected Case of Lemierre's Syndrome with Neck Abscess
}

\author{
Ippei Kishimoto $^{1)}$, Masahiro Kikuchi ${ }^{1)}$, Shogo Shinohara ${ }^{1)}$, Keizo Fujiwara ${ }^{1)}$, \\ Yuji Kanazawa ${ }^{1)}$, Risa Tona ${ }^{2)}$, Hiroyuki Harada ${ }^{1)}$ and Yasushi Naito ${ }^{12)}$
}

We report a suspected case of Lemierre's syndrome with a neck abscess. A 27-year-old man without any significant past medical history visited our hospital with a complaint of right neck pain which had lasted for three days. His tonsils were mildly infected and the uvula was shifted a bit toward the left side. He complained about severe pain in his right neck, which is not usually seen in cases of tonsillitis or peritonsillitis. He was treated with intravenous antibiotics for four days, however, his symptoms did not improve, and contrast-enhanced CT and MRI were performed. These images revealed the existence of a right parapharyngeal abscess and fresh thrombus in the right internal jugular vein extending into the lateral sinus. These findings suggested Lemierre's syndrome, but there was no abnormality on chest CT. Therefore, he was diagnosed as being suspected as having Lemierre's syndrome. After intraoral incisional drainage of the parapharyngeal abscess, his symptoms disappeared rapidly. Bacterial culture of the pus in the abscess showed growth of Fusobacterium necrophorum. Anticoagulant therapy with heparin and warfarin was also started and continued after discharge. On the 43rd day from onset of symptoms, remarkable reduction of thrombus was observed on the follow-up MRI. In this case, enhanced MRI was more useful than enhanced CT for detecting the presence of thrombus and confirming the extension of the thrombus. In examining patients with a peritonsillar abscess and severe neck pain, it is important to take Lemierre's syndrome into consideration because the disease may sometimes be life threatening if overlooked.

Keywords : Lemierre's syndrome, CT, MRI, anticoagulant therapy, neck pain

\section{References}

1) Karkos PD, Asrani S, Karkos CD, et al. : Lemierre's syndrome: A systematic review. Laryngoscope 119: 1552-1559, 2009.

2) Ng YY, Bellman S and Phelps PD : Computed tomography of earpits-deafness syndrome. Br J Radiol 62: 947-949, 1989.

3) Syed MI, Baring D, Addidle M, et al. : Lemierre syndrome: two cases and review. Laryngoscope 117: 1605-1610, 2007.

4) Weeks DF, Katz DS, Saxon P, et al. : Lemierre syndrome: report of five new cases and literature review. Emerg Radiol 17: 323-328, 2010.

5) Ridgway JM, Parikh DA, Wright R, et al. : Lemierre syndrome: a pediatric case series and review of literature. Am J Otolaryngol 31: 38-45, 2010

6) Aljohaney A and McCarthy A : Lemierre's syndrome with paradoxical emboli. Intern Med 49: 1433-1436, 2010.

7) Stallworth JR and Carroll JM : Lemierre's syndrome: new insights into an old disease. Clin Pediatr (Phila) 36: 715-717, 1997.
8) Sinave CP, Hardy GJ and Fardy PW : The Lemierre syndrome: suppurative thrombophlebitis of the internal jugular vein secondary to oropharyngeal infection. Medicine (Baltimore) 68: 85-94, 1989.

9) Wen $\mathrm{BC}$ and Dolan KD : Computed tomography of internal jugular vein thrombosis. Ann Otol Rhinol Laryngol 98: 318-319, 1989.

10) Tan ZL, Nagaraja TG and Chengappa MM : Fusobacterium necrophorum infections: virulence factors, pathogenic mechanism and control measures. Vet Res Commun 20: 113-140, 1996.

11) Hagelskjaer Kristensen L and Prag J : Human necrobacillosis, with emphasis on Lemierre's syndrome. Clin Infect Dis 31 : 524-532, 2000.

12) Lakshminarayana $\mathrm{PH}$ and Woodske ME : A unique case of lemierre syndrome associated with thrombophilia in an adult and the role of anticoagulation. Case Rep Med 2010: 982494, 2010.

13) Lu MD, Vasavada $Z$ and Tanner $C$ : Lemierre syndrome fol-

1) Department of Otolarygology, Kobe City Medical Center General Hospital

Corresponding Author Address : Ippei Kishimoto ippei5756jp@yahoo.co.jp

2) Department of Otolaryngology, Institute of Biomedical Research and Innovation Hospital 
lowing oropharyngeal infection: a case series. J Am Board Fam Med 22: 79-83, 2009.

14) Seo YT, Kim MJ, Kim JH, et al. : Lemierre syndrome: a case of postanginal sepsis. Korean J Intern Med 22: 211-214, 2007.

15) Goldhagen J, Alford BA, Prewitt LH, et al. : Suppurative thrombophlebitis of the internal jugular vein: report of three cases and review of the pediatric literature. Pediatr Infect Dis J 7: 410-414, 1988 .

16) Finegold SM, Bartlett JG, Chow AW, et al. : Management of anaerobic infections. Ann Intern Med 83: 375-389, 1975.

17) Dow SW : Management of anaerobic infections. Vet Clin North
Am Small Anim Pract 18: 1167-1182, 1988.

18) Chirinos JA, Lichtstein DM, Garcia J, et al. : The evolution of Lemierre syndrome: report of 2 cases and review of the literature. Medicine (Baltimore) 81: 458-465, 2002.

19) Dorton HE : Internal jugular vein thrombosis with fatal iatrogenic pulmonary embolism: a case report. Am Surg 41: 753754, 1975.

20) Williams A, Nagy M, Wingate J, et al. : Lemierre syndrome: a complication of acute pharyngitis. Int J Pediatr Otorhinolaryngol 45: 51-57, 1998.

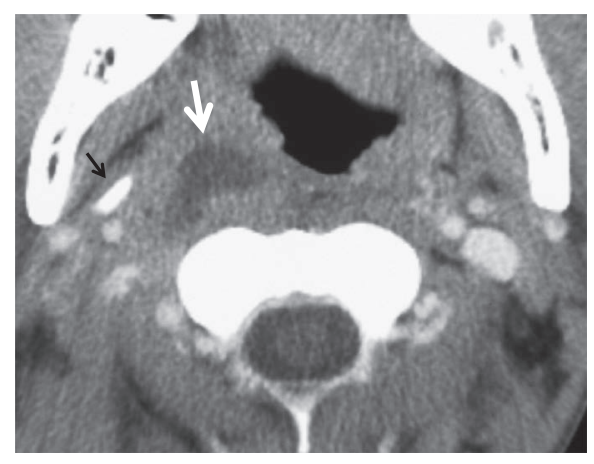

Contrast-enhanced axial CT scan of the neck on the fifth hospital day

The low density area in the right parapharyngeal space is more obvious than that of the second day (white arrow), and it was thought to be an abscess. The right internal jugular vein is not observed. The black arrow indicates the right styloid process.

a
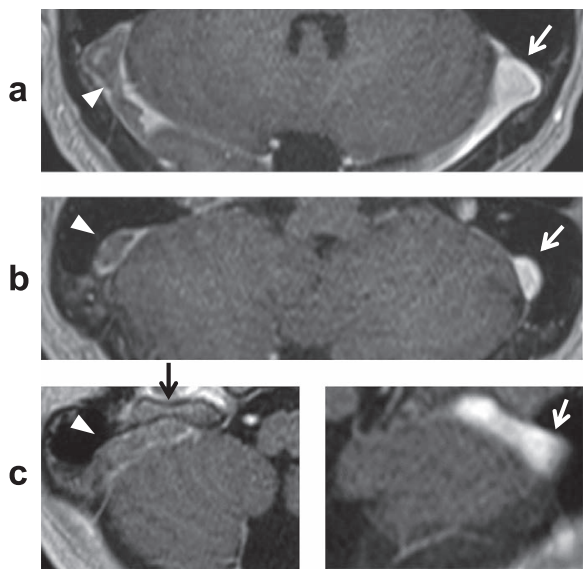

Gadolinium-enhanced axial MRI of the neck on the fifth hospital day

(a: transvers sinus level, b: from sigmoid sinus to transvers sinus level, c: sigmoid sinus level)

From the sigmoid sinus to transvers sinus, both of the vascular wall and intravascular lumen are enhanced on the left side (white arrow), while only the vascular wall was enhanced on the right side (white arrowhead). From these findings, thrombus formation was suspected. The black arrow indicates the bone marrow of the occipital bone.
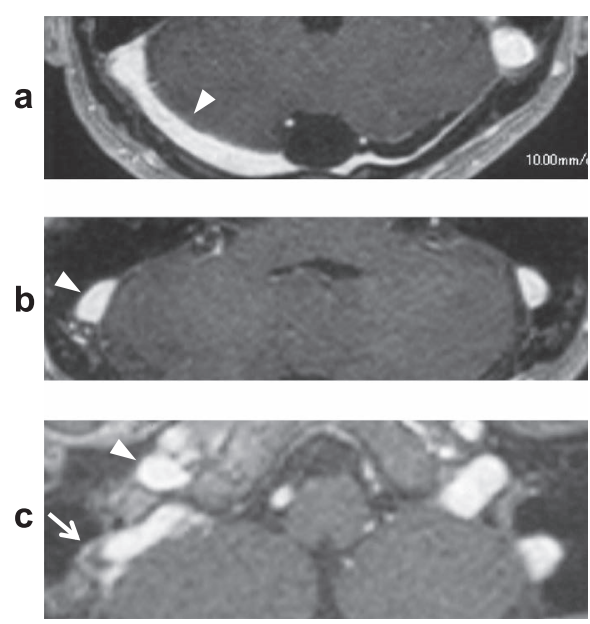

Gadolinium-enhanced axial MRI on the 43rd day from onset of symptom

(a: transvers sinus level, b: from sigmoid sinus to transvers sinus level, c: sigmoid sinus level)

The majority of the right internal jugular vein, transvers sinus and sigmoid sinus has been recanalized (arrow head), and a small residual thrombus can be observed only in the transition between the right sigmoid sinus and transvers sinus (arrow). 